\title{
A case of disseminated strongyloidiasis after multiple courses of immunosuppression
}

\author{
Jordan Mah MD, Anthony Lieu MD, Emma Holmes MD, Stephen Vaughan MD
}

Cite as: CMAJ 2022 January 24;194:E89-92. doi: 10.1503/cmaj.211056

A

71-year-old man presented to the emergency department with a 4-month history of weight loss, anorexia and constipation, and a 2-week history of abdominal pain.

He had biopsy-proven immunoglobulin G4 (IgG4)-related disease involving the pancreas and kidneys with 2 episodes of Gramnegative bacteremia over the past 3 years. The first episode was attributed to cholangitis and the second to a suspected obstructive pancreatic cancer. For these reasons, he underwent a cholecystectomy and Roux-en-Y hepaticojejunostomy; no cancer was found. During the previous 3 years, he had received multiple courses of immunosuppressive medications for flares of IgG4-related disease, including rituximab, mycophenolate mofetil and many courses of high-dose steroids (Figure 1). About 1 month before presentation, he had been started on high-dose steroids to treat a flare of IgG4related disease causing acute kidney injury. He had immigrated from China 40 years earlier and had not travelled recently.

\section{Key points}

- Strongyloides affects up to $40 \%$ of foreign-born Canadians and is often asymptomatic.

- Risk factors for hyperinfection, which is associated with a high risk of death, include use of immunosuppressant drugs (particularly corticosteroids), human T-cell leukemia virus type 1 infection and hematologic cancers.

- Strongyloides is diagnosed with serology and stool examination; sensitivity of these tests is decreased in patients who are immunosuppressed.

- All patients at risk of Strongyloides infection should have testing before beginning immunosuppressant drugs.

- Empiric ivermectin treatment should be offered to those at risk, if immunosuppression must be started before test results for Strongyloides are available.

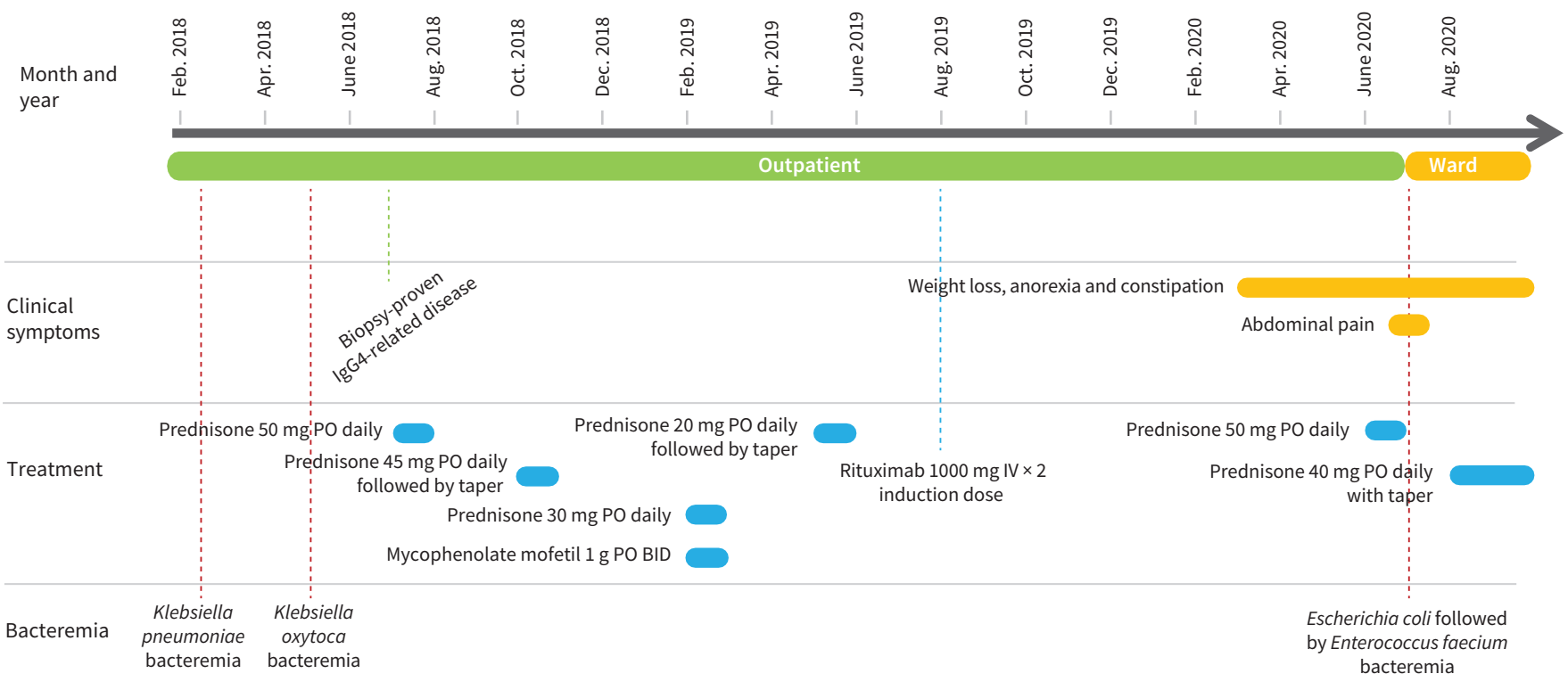

Figure 1: Timeline of diagnoses, bacteremia and immunosuppressive treatment of a 71-year-old patient with disseminated strongyloidiasis. Note: BID = twice daily, IGg4 = immunoglobulin G4, IV = intravenous, $\mathrm{PO}=$ orally. 
Table 1: Summary of laboratory investigations during admission

\begin{tabular}{|c|c|c|c|}
\hline Investigation & Presentation & 1 month earlier & Reference range \\
\hline Leukocyte $\left(10^{9} \mathrm{~g} / \mathrm{L}\right)$ & 5 & 10.4 & $4.0-11.0$ \\
\hline Absolute neutrophil count $\left(10^{9} \mathrm{~g} / \mathrm{L}\right)$ & 4.6 & 7.2 & $2.0-8.0$ \\
\hline Absolute lymphocyte count $\left(10^{9} \mathrm{~g} / \mathrm{L}\right)$ & 0.2 & 1.1 & $0.7-3.5$ \\
\hline Absolute eosinophil count $\left(10^{9} \mathrm{~g} / \mathrm{L}\right)$ & 0 & 0.6 & $0.0-0.7$ \\
\hline Hemoglobin (g/L) & 67 & 91 & $137-180$ \\
\hline Platelets $\left(10^{9} \mathrm{~g} / \mathrm{L}\right)$ & 93 & 270 & $150-400$ \\
\hline C-reactive protein (mg/L) & 112.1 & 35.4 & $0-8$ \\
\hline Ferritin $(\mu \mathrm{g} / \mathrm{L})$ & 906 & 440 & $30-500$ \\
\hline Sodium (mmol/L) & 128 & 133 & $133-145$ \\
\hline Albumin (g/L) & 25 & 30 & $35-45$ \\
\hline Alanine aminotransferase (U/L) & 32 & 61 & $1-60$ \\
\hline Y-Glutamyl transferase (U/L) & 248 & 173 & $11-62$ \\
\hline Alkaline phosphatase (U/L) & 294 & 190 & $30-145$ \\
\hline Creatinine $(\mu \mathrm{mol} / \mathrm{L})$ & 164 & 204 & $50-120$ \\
\hline
\end{tabular}

When we examined the patient, he was afebrile and hemodynamically stable. He was cachectic, but his physical examination was otherwise unremarkable. Laboratory investigations are shown in Table 1.

Blood cultures drawn on admission and they grew Escherichia coli. We started intravenous ertapenem, and subsequent blood cultures were sterile. A computed tomography scan of the patient's chest and abdomen showed extensive bilateral parenchymal lung abnormalities that had not been seen on previous scans, dilated stomach and small bowel loops, with no evidence of mechanical obstruction, and no potential focus of infection (Figure 2 and Figure 3). Upper and lower endoscopies were normal. We diagnosed gastroparesis and started the patient on motility agents.

A week later, he developed Enterococcus faecium bacteremia, which we treated with intravenous vancomycin. The infectious disease service raised the possibility of Strongyloides stercoralis hyperinfection syndrome (SHS). However, he had received numerous immunosuppressive drugs previously

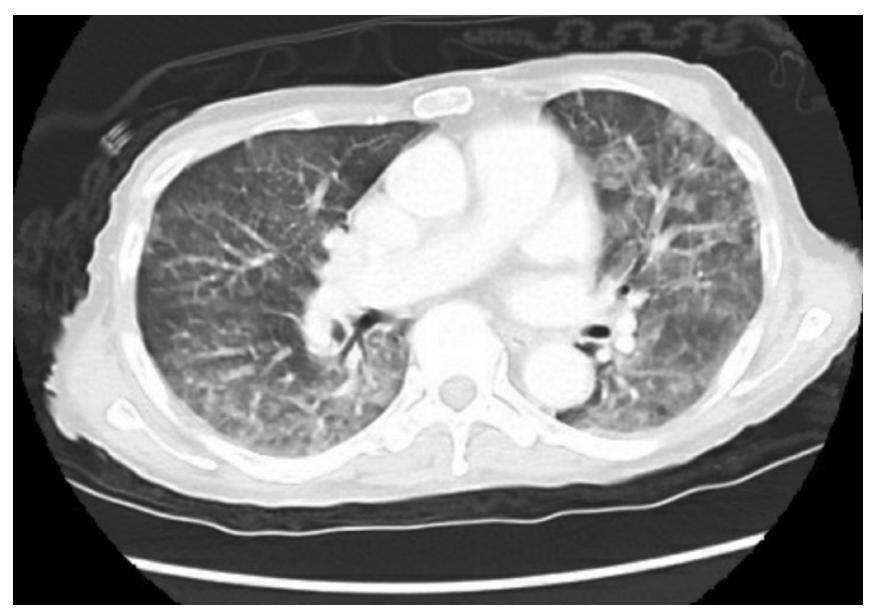

Figure 2: Computed tomography scan of the patient's chest at admission, showing bilateral patchy infiltrates. and had not developed SHS, so we thought the syndrome was unlikely. The National Reference Centre for Parasitology in Canada tested for S. stercoralis serology (NIE enzyme-linked immunoassay) and the result was negative. We could not send stool for microscopy owing to the patient's constipation.

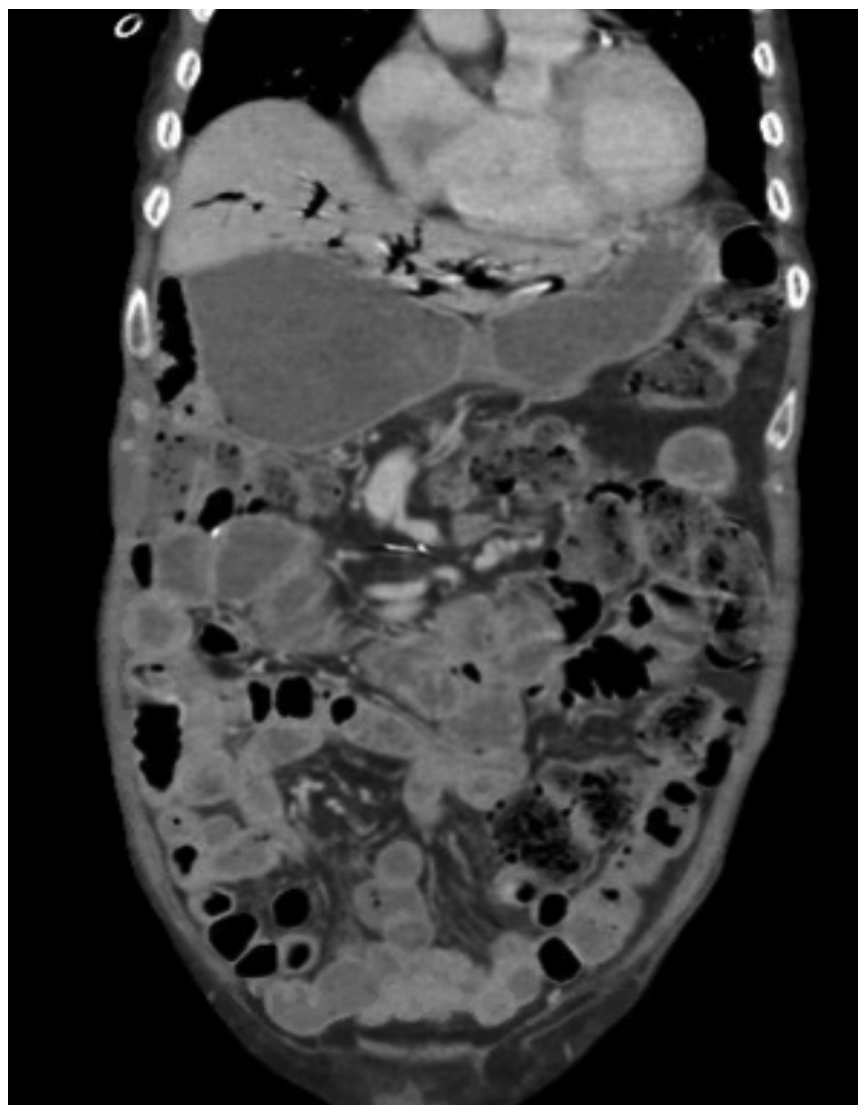

Figure 3: Computed tomography scan of the patient's abdomen at admission, showing dilated stomach and small bowel loops without evidence of a high-grade mechanical obstruction. 
Thirteen days after admission, he developed septic shock and died from a suspected aspiration pneumonia. A limited autopsy showed multiple roundworms throughout his stomach, small bowel and pancreas (Figure 4). The presence of parasites in the pancreas confirmed disseminated strongyloidiasis.

\section{Discussion}

\section{Epidemiology of Strongyloides}

Strongyloides stercoralis is an intestinal nematode that can cause an asymptomatic chronic gastrointestinal infection that can remain quiescent for years. ${ }^{1,2}$ It is endemic to Africa, Asia and Central and South America; about 30 million100 million people are affected worldwide. ${ }^{1-3}$ It is estimated that $10 \%-40 \%$ of the population are affected in endemic regions. In Canada, 2.5 million individuals are thought to be infected with Strongyloides. ${ }^{1-3}$

Strongyloides completes its entire life cycle in humans, with the potential for autoinfection. ${ }^{1-3}$ Overamplification of the parasitic load at a rate that escapes the host's ability to control the infection is known as hyperinfection; dissemination refers to the presence of parasites in organs not typically involved in the parasite's life cycle, such as the pancreas. ${ }^{1-3}$ Immunosuppression, particularly suppression of cell-mediated immunity, can trigger SHS or dissemination, with mortality rates of $85 \%-100 \% .{ }^{1,4}$ Corticosteroid administration is a well-described risk factor for SHS; even short steroid courses of 5 days are associated with SHS and death. ${ }^{1,2,4}$ During SHS, the parasitic burden increases and mature larvae penetrate the bowel mucosa and enter the host's circulation, where they can disseminate to other organs. ${ }^{1}$

\section{Who should be screened for Strongyloides?}

The Committee to Advise on Tropical Medicine and Travel (CATMAT), an advisory body to the Public Health Agency of Canada, recommends screening all individuals at high risk for developing Strongyloides hyperinfection, based on their epidemiologic exposure

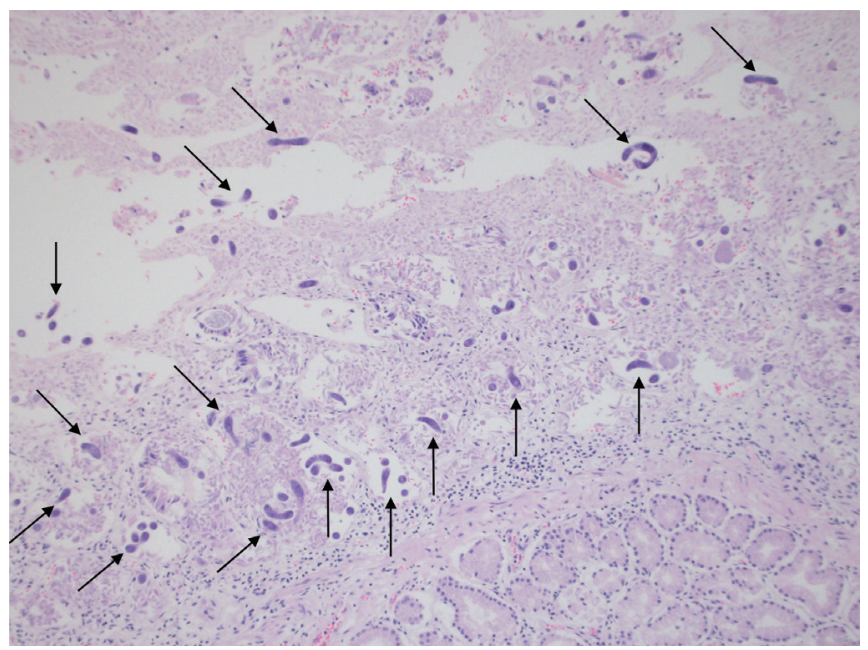

Figure 4: Hematoxylin and eosin stain of the gastrointestinal tract under 40x magnification. Multiple roundworms, measuring 350-600 × $15 \mu \mathrm{m}$, can be seen (arrows) in the lumen of the small bowel. and clinical risk factors for dissemination. ${ }^{3}$ Risk factors for Strongyloides infection include birth, residence or long-term travel (> 6 months) in Southeast Asia, Oceania, sub-Saharan Africa, South America or the Caribbean, although infection can occur with shorter periods of exposure if the person engages in highrisk activities. ${ }^{3}$ Because Strongyloides is associated with fecal contamination of water or soil, high-risk activities include residing in rural or beach settings and skin contact with sand or soil. ${ }^{3}$ Clinical risk factors associated with SHS include human T-cell leukemia virus type 1 (HTLV-1) infection, treatment with glucocorticoid or immunomodulatory agents, organ transplantation and hematologic malignancies. ${ }^{3}$

The presence of unexplained serum eosinophilia in a patient who was born in or has travelled to an endemic region should prompt investigation for Strongyloides; however, its absence does not rule out infection. ${ }^{5}$ Screening for S. stercoralis should occur before the administration of immunosuppressive therapy, transplantation, or in patients with other immunosuppressive conditions. ${ }^{1-3,5}$ The widespread use of corticosteroids for the treatment of patients with COVID-19 has led the Ontario COVID19 Science Advisory Table to recommend serologic testing for S. stercoralis in patients with COVID-19 who have moderate to high epidemiologic risk. ${ }^{6}$

\section{Diagnostic tests for Strongyloides}

The sensitivity of stool microscopy is poor; a single stool examination fails to identify larvae in up to $70 \%$ of cases, owing to intermittent shedding and low larval burden. ${ }^{1-4}$ However, the diagnostic yield of stool microscopy improves to more than $95 \%$ if 7 serial samples are examined or if a stool agar culture is performed..$^{1-4}$ Serologic testing is the most sensitive method for diagnosing chronic strongyloidiasis in patients who are immunocompetent, as the sensitivity of commercial and in-house assays ranges from $83 \%$ to $95 \% .^{3,5,7}$ In patients with HTLV-1 infection or hematologic cancers or who have received immunosuppressive medications, the sensitivity decreases to $42.9 \%$, owing to a blunted immune response. ${ }^{8}$ Both stool microscopy and serologic testing are recommended in these patients. ${ }^{3,5}$

In patients with SHS, the diagnostic yield increases, because larvae are more easily identified in duodenal aspirate, sputum, bronchoalveolar lavage fluid or stool samples. ${ }^{1}$ To establish a diagnosis during SHS, the following are suggested: 3 serial stool samples for ova and parasite, sputum ova and parasite examination for S. stercoralis, S. stercoralis serology, blood culture looking for enteric organisms, and biopsy of organs where S. stercoralis involvement is suspected. ${ }^{4}$

\section{Treatment of strongyloidiasis}

CATMAT Guidelines recommend treatment of chronic, asymptomatic strongyloidiasis with oral ivermectin, $200 \mu \mathrm{g} / \mathrm{kg} / \mathrm{d}$ given in 2 doses on consecutive days, or 14 days apart. ${ }^{3}$ This is well tolerated and associated with high cure rates, and when there is no ivermectin shortage, treatment should be given to all immunocompetent individuals with known asymptomatic infection. ${ }^{1,5}$ More recently, a single dose of ivermectin has been shown to be as effective as 2 doses. $^{9}$ 
For patients with risk factors for Strongyloides who require immediate immunosuppression before test results for Strongyloides are available, empiric treatment with ivermectin is recommended. ${ }^{3,5}$ This applies to patients with COVID-19 who are about to be treated with corticosteroids. When serology results are indeterminate, the Ontario COVID-19 Science Advisory Table recommends treatment., ${ }^{6,10}$

Patients with suspected Strongyloides infection from countries in Central Africa, such as Congo, Cameroon and Central African Republic, should be referred to a tropical medicine specialist to exclude loiasis co-infection, as ivermectin can precipitate life-threatening encephalopathy in patients with high levels of microfilaria from loiasis. ${ }^{3,5}$

In cases of suspected SHS or dissemination, empiric dual therapy with ivermectin and albendazole is recommended while awaiting the results of diagnostic tests. ${ }^{3,5}$ This is based on expert opinion and clinical experience, given the substantial mortality associated with this condition. ${ }^{3,5}$

\section{Case revisited}

Our patient presented several challenges to the diagnosis of SHS, which may have contributed to his diagnosis being made post mortem. He presented with nonspecific gastrointestinal symptoms that we attributed to gastroparesis. Dissemination or SHS can present with a wide range of clinical symptoms and signs, with no pathognomonic feature. ${ }^{1,3,4}$ Respiratory and gastrointestinal symptoms are common but nonspecific and include dyspnea, wheezing, acute respiratory failure, abdominal pain, anorexia, weight loss and gastrointestinal bleeding. ${ }^{1}$ Our patient's pulmonary symptoms and extensive bilateral infiltrates were likely explained by larval migration through the lung during SHS. Second, while our patient had recurrent episodes of Gramnegative bacteremia, there was always a plausible source: cholangitis and suspected pancreatic cancer. In retrospect, the bacteremia may have been caused by Strongyloides. Recurrent, unexplained Gram-negative sepsis or meningitis should raise the possibility of Strongyloides, as enteric organisms adhere to the parasite during migration into tissue. ${ }^{1,2,4}$ For the same reason, bacteremia with $E$. faecalis, a common commensal of the human gastrointestinal tract, is described in SHS or dissemination. ${ }^{11}$ Of note, no roundworms were identified when the previous surgical pathology slides from the patient's Roux-en-Y procedure and cholecystectomy were re-examined.
During our patient's last admission to hospital, his serologic test was negative, reflecting the test's poor sensitivity in immunocompromised hosts. ${ }^{8}$ Our case highlights that the diagnosis of disseminated strongyloidiasis is often delayed, leading to involvement of many organs with significant risk of mortality.

\section{Conclusion}

Patients at risk of Strongyloides infection should be screened before immunosuppression, as a substantial proportion of Canada's population is at risk. The development of complications including SHS and dissemination are associated with high mortality.

\section{References}

1. Krolewiecki A, Nutman TB. Strongyloidiasis: a neglected tropical disease. Infect Dis Clin North Am 2019;33:135-51.

2. Schär F, Trostdorf U, Giardina F, et al. Strongyloides stercoralis: global distribution and risk factors. PLoS Negl Trop Dis 2013;7:e2288. doi: 10.1371/journal. pntd.0002288.

3. Boggild AK, Libman M, Greenaway C, et al. CATMAT statement on disseminated strongyloidiasis: prevention, assessment and management guidelines. Can Commun Dis Rep 2016;42:12-9.

4. Lim S, Katz K, Krajden S, et al. Complicated and fatal Strongyloides infection in Canadians: risk factors, diagnosis and management. CMAJ 2004;171:479-84.

5. Requena-Méndez A, Buonfrate D, Gomez-Junyent J, et al. Evidence-based guidelines for screening and management of strongyloidiasis in non-endemic countries. Am J Trop Med Hyg 2017;97:645-52.

6. Leung E, Mclntyre M, Andany N, et al.; Ontario COVID-19 Science Advisory Table and the Drugs \& Biologics Clinical Practice Guidelines Working Group. Ivermectin to prevent disseminated Strongyloides infection in patients with COVID-19. Science Briefs of the Ontario COVID-19 Science Advisory Table 2021;2:1-7.

7. Bisoffi Z, Buonfrate D, Sequi M, et al. Diagnostic accuracy of five serologic tests for Strongyloides stercoralis infection. PLoS Negl Trop Dis 2014;8:e2640. doi: 10.1371/journal.pntd.0002640.

8. Luvira V, Trakulhun K, Mungthin M, et al. Comparative diagnosis of strongyloidiasis in immunocompromised patients. Am J Trop Med Hyg 2016;95:401-4.

9. Buonfrate D, Salas-Coronas J, Muñoz J, et al. Multiple-dose versus single-dose ivermectin for Strongyloides stercoralis infection (Strong Treat 1 to 4): a multicentre, open-label, phase 3, randomised controlled superiority trial. Lancet Infect Dis 2019;19:1181-90.

10. Stauffer WM, Alpern JD, Walker PF. COVID-19 and dexamethasone: a potential strategy to avoid steroid-related Strongyloides hyperinfection. JAMA 2020;324:623-4.

11. Wee LE, Hnin SWK, Xu Z, et al. Strongyloides hyperinfection associated with Enterococcus faecalis bacteremia, meningitis, ventriculitis and gas-forming spondylodiscitis: a case report. Trop Med Infect Dis 2020;5:44.
Competing interests: Stephen Vaughan reports receiving payment or honoraria from GSK. No other competing interests were declared.

This article has been peer reviewed.

The authors have obtained patient consent.

Affiliations: Division of Infectious Diseases, Department of Medicine (Mah, Lieu, Vaughan); Department of Pathology and Laboratory Medicine (Holmes), University of Calgary, Calgary, Alta.
Contributors: Jordan Mah and Anthony Lieu contributed to the conception and design of the work and drafted the manuscript. All authors critically revised the manuscript for important intellectual content, gave final approval of the version to be published and agreed to be accountable for all aspects of the work.

Content licence: This is an Open Access article distributed in accordance with the terms of the Creative Commons Attribution
(CC BY-NC-ND 4.0) licence, which permits use, distribution and reproduction in any medium, provided that the original publication is properly cited, the use is noncommercial (i.e., research or educational use), and no modifications or adaptations are made. See: https://creativecommons.org/licenses/ by-nc-nd/4.0/

Correspondence to: Jordan Mah, jordan.mah@albertahealthservices.ca 